\title{
Environmental Effect of Ambient Charge in a Cournot Oligopoly Market
}

\author{
Akio Matsumoto ${ }^{1, a,{ }^{*}}$ and Ferenc Szidarovszky ${ }^{2, b}$ \\ ${ }^{1}$ Department of Economics, Chuo University, 742-1, Higashi-Nakano, Hachioji, \\ Tokyo, Japan \\ ${ }^{2}$ Department of Applied Mathematics, University of Pecs, Pecs, Injusag u-6, Hungary \\ aakiom@tamacc.chuo-u.ac.jp, ${ }^{b}$ szidarka@gmail.come
}

Keywords: Ambient Charge, Nonpoint source (NPS) pollutions, Cournot competition.

\begin{abstract}
This paper investigates the effect caused by an increase in ambient charges on firm-specific and total pollutions in a Cournot oligopoly market. Formalizing profit-maximizing behavior in the n-firm framework with product differentiation, we show that ambient change can reduce industrial pollutions.
\end{abstract}

\section{Introduction}

This paper investigates the effects caused by ambient charges on the pollutions arose in an n-firm Cournot market. Its attention is put on nonpoint source (NPS) pollution such as water and air pollutions from diffuse sources. Although any pollutant originates from a single point source, the long-range transport ability and multiple sources of the pollutant make it a nonpoint source of pollution. For the government that would like to control pollutions, it might be impossible to measure firm-specific emissions whereas possible to measure the total level of pollution. As suggested by Segerson [3], the government adopts such an environmental policy to establish a cut-off level of the whole pollution and to make the following rule: regardless of firm specific emission level, if the actual level of the total pollutions exceeds the cut-off level, then all firms levy the same penalty while if the actual level falls short of the cut-off level, then all firms are awarded the same subsidy. Ganguli and Raju [1] show a "perverse" ambient charge effect on total pollution, an increase in the ambient charge could lead to greater pollution, in the Bertrand duopoly. On the other hand, Raju and Ganguli [2] examine the same subject in a Cournot duopoly framework and attain the effective result such that an increase in the ambient charges reduce pollutant emissions. This study steps forward and demonstrate the effective environmental policy in an n-firm Cournot market.

This paper is organized as follows. Section 2 presents the basic mathematic model. Section 3 considers the effect of increasing ambient charges on individual as well as total pollutions. Section 4 concludes the paper..

\section{Cournot Model}

There are $n$ firms in the oligopoly market, producing a differentiated product. The production quantity and price of firm $k$ are represented by $q_{k}$ and $p_{k}$. It is assumed that the linear price function of good $\mathrm{k}$ is 


$$
p_{k}=\alpha-q_{k}-\gamma \sum_{i \neq k}^{n} q_{i}, k=1,2, \ldots, n
$$

where $n \geq 2$ and $0<\gamma<1$, implying that the goods are substitutes. It is also assumed that all the firms have the same production cost, $c$. Firm $\mathrm{k}$ emits pollutants $e_{k} q_{k}$ in connection with its production with $e_{k}>0$. The government can measure a total emission quantity and has an exogenously determined environmental standard E. According to $m$ times the difference between the total emission, $\sum_{k=1}^{n} e_{k} q_{k}$ and the standard, it will levy the penalty if the difference is positive and award the subsidy if negative. The profit of firm $\mathrm{k}$ is expressed as

$$
\pi_{k}=\left(p_{k}-c\right) q_{k}-m\left(\sum_{i=1}^{n} e_{i} q_{i}-\bar{E}\right)
$$

with the marginal cost of firm $\mathrm{k}$ is $c_{k}=c+m e_{k}$. To avoid negative optimal production, we assume a large enough value of $\alpha$ so that $\alpha-c_{k}>0$ holds for all $k$. It can be easily checked that the second-order condition is certainly satisfied. The Cournot equilibrium output for firm $k$ is obtained by solving the following simultaneous equations:

$$
q_{k}+\frac{\gamma}{2} \sum_{i \neq k}^{n} q_{i}=\frac{\alpha-c_{i}}{2}
$$

or in vector form $B q=A$ where

$$
q=\left(q_{i}\right), A=\left(\frac{\alpha-c_{i}}{2}\right), B=\left(B_{i j}\right) \text { with } B_{i i}=1 \text { and } B_{i j}=\frac{\gamma}{2}
$$

Since B is invertible, the Cournot output vector is given by $q=B^{-1} A$ where the diagonal and off-diagonal elements of $B^{-1}$ are, respectively,

$$
\frac{2(2+(n-2) \gamma)}{(2-\gamma)(2+(n-1) \gamma)} \text { and }-\frac{2 \gamma}{(2-\gamma)(2+(n-1) \gamma)}
$$

Hence the Cournot equilibrium output of firm $k$ with $c_{k}=c+m e_{k}$ is

$$
q_{k}^{C}=\frac{\left(\alpha-c_{k}\right)(2-\gamma)+m\left[\gamma \sum_{i=1}^{n} e_{i}-(2+(n-1) \gamma) e_{k}\right]}{(2-\gamma)(2+(n-1) \gamma)}
$$

\section{Ambient Charge}

In this section we examine the effect of a change in the ambient charge on firm-specific production and then total pollutions. Differentiating $q_{k}^{C}$ with respect to m yields

$$
\frac{\partial q_{k}^{C}}{\partial m}=\frac{\gamma \sum_{i=1}^{n} e_{i}-(2+(n-1) \gamma) e_{k}}{(2-\gamma)(2+(n-1) \gamma)}
$$

Where the numerator can be written as 


$$
n \gamma\left(\bar{e}-e_{k}\right)-(2-\gamma) e_{k} \text { with } \bar{e}=\frac{1}{n} \sum_{i=1}^{n} e_{i}
$$

It is definitely negative if $\bar{e} \leq e_{k}$, that is, firm $k$ is higher-or average-polluted. The sign seems to be ambiguous if $\bar{e}>e_{k}$. However, since the numerator is differently expressed as

$$
n \gamma\left(\bar{e}-\tilde{e}_{k}\right) \text { with } \tilde{e}_{k}=\frac{n \gamma+(2-\gamma)}{n \gamma} e_{k}
$$

changing $m$ has a negative effect if $\bar{e}<\tilde{e}_{k}$ and a positive effect if $\bar{e}>\tilde{e}_{k}$. Hence summarizing the results on firm-specific production levels gives the following.

Theorem 1. An increase in the ambient charge decreases production of higher- or average-polluted firm whereas it decreases, increases or does not change production of lower-polluted firm $k$ according to whether $\bar{e}>\tilde{e}_{k} \bar{e}<\tilde{e}_{k}$ or $\bar{e}=\tilde{e}_{k}$.

Concerning the total quantity of emission at the Cournot equilibrium,

$$
E^{C}=\sum_{k=1}^{n} e_{k} q_{k}^{C}
$$

We have the following result:

Theorem 2. Given positive $e_{k}>0$ for all $k$, then increasing the policy parameter $m$ decreases the total emission, $E^{C}$.

Proof: Differentiating $E^{C}$ with respect to $\mathrm{m}$ and arranging the terms give

$$
\frac{\partial E^{C}}{\partial m}=\frac{\gamma\left(\sum_{k=1}^{n} e_{k}\right)^{2}-(2+(n-1) \gamma) \sum_{k=1}^{n} e_{k}^{2}}{(2-\gamma)(2+(n-1) \gamma)}
$$

The denominator is definitely positive. Let us denote the numerator by $\mathrm{S}(\gamma)$,

$$
S(\gamma)=\gamma\left(\sum_{k=1}^{n} e_{k}\right)^{2}-(2+(n-1) \gamma) \sum_{k=1}^{n} e_{k}^{2}
$$

We then obtain the trivial result

$$
S(0)=-2 \sum_{k=1}^{n} e_{k}^{2}<0
$$

and

$$
S(1)=\left(\sum_{k=1}^{n} e_{k}\right)^{2}-(1+n) \sum_{k=1}^{n} e_{k}^{2}
$$

where the direction of the inequality is shown as follows. The Cauchy inequality implies

$$
\left(\sum_{k=1}^{n} e_{k} \quad 1\right)^{2} \leq \sum_{k=1}^{n} e_{k}^{2} \sum_{k=1}^{n} 1
$$

so

$$
\left(\sum_{k=1}^{n} e_{k} \quad 1\right)^{2} \leq n \sum_{k=1}^{n} e_{k}^{2}
$$


Using the last inequality above we can arrive at $S(1)<0$ because

$$
S(1)=\left(\sum_{k=1}^{n} e_{k}\right)^{2}-(1+n) \sum_{k=1}^{n} e_{k}^{2}<\left(\sum_{k=1}^{n} e_{k}\right)^{2}-n \sum_{k=1}^{n} e_{k}^{2} \leq 0
$$

Since $S(\gamma)$ is linear in $\gamma$ and both $S(0)$ and $S(1)$ are negative, the value of $S(\gamma)$ is also negative for any $\gamma \in(0,1)$. Therefore, we have the negative derivative. Q.E.D.

\section{Concluding Remarks}

The effect of the ambient charges on total pollution in a Cournot oligopoly was examined. First the Cournot equilibrium was determined. An increase of the ambient charge can have a diverse effect on the production levels: if a firm is higher- or average-polluted, then its output decreases, otherwise it can increase, decrease or remain the same depending on a simple condition derived in the paper. It is also proved that the total emission decreases by increasing the policy parameter.

\section{References}

[1] Ganguli, S. and S. Raju, Perverse environmental effects of ambient charges in a Bertrand duopoly, Journal of Environmental Economics and Policy, 1 (2012), 1-8.

[2] Raju S. and S. Ganguli, Strategic firm interaction, returns to scale, environmental regulation and ambient charges in a Cournot duopoly, Technology and Investment, 4 (, 2013), 113-122,

[3] Segerson, K., Uncertainty and incentives for non-point pollution control, Journal of Environmental Economics and Management, 15 (1988), 87-98. 